\title{
The Role of Nanoparticle PEGylation in Drug Delivery
}

\author{
Ellina A. Mun ${ }^{1 *}$, Balnur A. Zhaisanbayeva ${ }^{2}$, \\ ${ }^{1}$ National Center for Biotechnology, $13 / 5$ Qorgalzhyn highway, Nur-Sultan, 010000 Kazakhstan; \\ 2 School of Engineering, Nazarbayev University, 53 Qabanbay Batyr Ave, Nur-Sultan, 010000 Kazakhstan \\ * Corresponding author. Tel.:+7 70221088 77.Email: ellina.moon@gmail.com
}

\begin{abstract}
Over the past few decades, nanoparticles have been attracting significant attention of researches in chemical, biomedical, pharmaceutical sciences, due to their unique physicochemical properties. This includes ultra small size, large surface area, good biocompatibility and high reactivity. In particular, nanoparticles are promising for pharmaceutical and biomedical fields, as they have been applied as drug carriers and diagnostic tools. However, nanoparticles can be easily detected and cleared out by the mononuclear phagocyte system before delivering the drug to the target site. One of the most widely applied approaches to prolong circulation of nanoparticles is to modify their surfaces with polyethylene glycol (PEG). This paper describes how PEGylation occurs, as well as application of various PEGylated nanoparticles in drug delivery.
\end{abstract}

\section{Keywords}

Nanoparticles; PEGylation; drug delivery; mononuclear phagocyte system.

(C) Ellina A. Mun, Balnur A. Zhaisanbayeva, 2020

\section{Introduction}

Nanoparticles (NPs) have a significant potential for drug delivery due to their unique physicochemical properties, including their ultra small size, high reactivity and large surface area to mass ratio which can offer significant benefits compared to traditional therapeutic and diagnostic agents [1]. Due to these reasons, nanoparticles have been attracting a considerable interest in biomedical and pharmaceutical sciences over the last two decades. They have been successfully applied as drug carriers $[2,3]$, diagnostic tools $[4,5]$, labelling and tracking agents $[6,7]$. A large group of inorganic nanomaterials for biomedical application have been described, including gold, titanium, iron oxide and silica. Whilst gold has been widely explored and has a long history of use, silica nanoparticles are less well defined but appear promising and are the subject of recent investigations for drug delivery [8]. Silica nanoparticles are inexpensive and easy to prepare and separate, safe and biocompatible, also their surfaces can be easily functionalised, and thus have an ongoing role in both in vitro and in vivo biomedical nanotechnology [9].
The advantages of using nanoparticles for drug delivery include fine control over particle size [10-13], site-specific targeting $[14,15]$, the potential for surface modification [16, 17], controlled and sustained release of the drug [18-20] and the potential to use different routes of drug administration. However, there are some significant limitations, including the possibility that nanoparticles can be easily detected by the immune system once in the blood stream, and may be cleared by the mononuclear phagocyte system (MPS) (liver, spleen, lungs and bone narrow) via opsonisation (a binding enhancer for the process of phagocytosis) before they can deliver the drug to the target site $[21,22]$. Opsonins are the blood serum components involved in the phagocytic recognition process, the most common of which are $\mathrm{C} 3, \mathrm{C} 4, \mathrm{C} 5$ proteins and immunoglobulins [22].

\section{How does PEGylation work?}

There are two main approaches to prolong the circulation of nanoparticles and to minimise opsonisation; surface functionalisation of nanoparticles with hydrophilic polymers/surfactants and/or synthesis 
of nanoparticles using biodegradable copolymers with hydrophilic segments (polyethylene glycol, poloxamer, poloxamine and polysorbate 80) [21]. Among those, the most widely used strategy is surface modification of nanoparticles with polyethylene glycol (PEG) [23], which shows low adverse effects in vivo [22] and is approved for human intravenous administration [24]. Grafting or adsorption of PEG or PEG-containing copolymers to the surface of nanoparticles prolongs blood circulation half-life up to several orders of magnitude and provides a hydrophilic protective layer which limits recognition by opsonin proteins due to steric repulsion forces [22]. PEG surface coverage can occur in "mushroom" and "brush" configurations. The first is observed at low surface chain densities where PEG molecules are located close to the surface of nanoparticles and have a broad range of motions. At higher surface coverage, PEG chains are in a semilinear configuration and their range of motion is significantly restricted ("brush" configuration). The optimal surface coverage is between the "mushroom" and "brush" configurations allowing the PEG density to be high enough to avoid gaps in the PEG protective layer whilst PEG chains are mobile [21, 22].

PEG-containing polymers can be either adsorbed or covalently attached to the surface of nanoparticles but both of these approaches have limitations. The first method is simple to achieve but adsorbed PEG polymers can desorb leaving some areas of nanoparticle surface uncovered and available for opsonins to bind. When PEG molecules are covalently attached, it may be difficult to determine whether they are on the surface of the NPs, or in the bulk and it is difficult to control and optimise the coverage density and PEG conformation. However, covalent surface modification with PEG is usually the preferred approach for biodegradable nanoparticles. In the synthesis of biodegradable NPs, PEG is typically used with poly(lactic acid), poly(lactic acid-co-glycolic acid), and poly(alkylcyanoacrylates) [22].

\section{PEGylation of different nanoparticles}

PEGylation is widely used to optimise the surface properties of various types of nanoparticles, including gold nanoparticles which are reported to be nontoxic [25]. Apart from preventing them from being recognised by phagocytes system, PEGylation of gold nanoparticles enhances their solubility and stability under physiological conditions and enables further surface modification via coupling reactions [26]. It was demonstrated that both laser-generated and chemically synthesised gold nanoparticles (NPs) can be PEGylated through covalent attachment of thiol-bearing PEG chains, but the former ones exhibit improved stability and controllable surface coverage [26].

PEGylated NPs can be efficient drug delivery platforms, as PEG provides water-soluble and biocompatible cage-type protection for hydrophobic drug molecules and allows their delivery to the target site, as demonstrated by Cheng and coworkers with PEGylated gold NPs-silicon phthalocyanine 4 (Pc4) conjugates. The cage-type protection caused by steric repulsion leads to separation of Au NPs, stabilisation of Pc4 due to van der Waals interactions and protects the drug from aqueous media. Drug delivery time to the target tumor was significantly reduced to 2 hours with PEGylated Au NP-Pc 4 conjugates, compared to 2 days for the free drug [24]. PEGylation can also convert the hydrophobic nanoparticle surface into a hydrophilic one acting as a linker and allowing further functionalisation with the drug molecules, improving biocompatibility and resulting in superior ability to penetrate the cell nucleus compared to the drug alone [27].

In contrast to gold nanoparticles which can be PEGylated through surface functionalisation, PEGylated polymer nanoparticles are usually synthesised from PEG containing block-copolymers $[28,29]$. For example, poly(e-caprolactone)/poly (ethylene glycol) (PCL/PEG) nanoparticles were developed from a PCL/PEG/PCL triblock copolymer by a solvent extraction method using acetone [29]. The PCL/PEG/PCL copolymer was synthesised by ring-opening polymerisation of $\varepsilon$-caprolactone using PEG as a macroinitiator. PCL/PEG/PCL NPs were loaded with doxorubicin, a potent anticancer agent, by a pH-induced self-assembly method with $91.7 \%$ encapsulation efficiency. Improving doxorubicin delivery and safety is important since it is a highly cytotoxic compound that can damage normal tissue before reaching the target tumor. Doxorubicin loaded PCL/PEG/PCL nanoparticles were found to be a promising delivery system owing to their ability to release doxorubicin more quickly at $\mathrm{pH} 5.5$ than at $\mathrm{pH}$ 7.0 , which allows the drug to be only slowly released in the plasma $(\mathrm{pH}=7.4)$ but rapidly released at the solid tumor site $(\mathrm{pH}=5.5)$. In vitro studies demonstrated reduced cytotoxicity of doxorubicin encapsulated in PCL/PEG/PCL nanoparticles in the C-26 cell line and doxorubicin in PCL/PEG/PCL nanoparticles was shown to treat mice bearing subcutaneous C-26 tumors more efficiently than the free drug [29]. 
For drug delivery to mucosal epithelia, PEGylation is a useful approach for enhancing drug transport through mucus by reducing the interactions of a nanoparticulate carrier with the mucus layer, which is a physical and enzymatic barrier protecting epithelial cells of the human body [30-32]. Conventional nanoparticles, once administrated to mucosal tissues, are trapped in mucus by steric or adhesive forces and then eliminated by mucociliary clearance, whereas PEGylation enables the particles to overcome this barrier and penetrate into the mucus layer [31]. Lai and coworkers studied the diffusion of PEG-modified polystyrene nanoparticles in fresh undiluted human cervical mucus. They found that a dense coating with low molecular weight $(2 \mathrm{kDa})$ PEG enhanced the transport of nanoparticles $(100-500 \mathrm{~nm})$ by up to 3 orders of magnitude, which correlated with a decreased number of particles immobilised by the mucus (less than $0.5 \%$ for PEGylated versus 32-45\% for uncoated nanoparticles). The study also showed that both low molecular weight PEG and a high density of surface coverage were crucial for PEG-modified nanoparticles to rapidly penetrate through the mucus [31]. In addition, polystyrene PEGylated nanoparticles were able to penetrate through the highly viscoelastic mucus layer which is a challenge in the treatment of chronic rhinosinusitis [32]. $\mathrm{Cu}$ and Saltzman [30] demonstrated that the addition of PEG of different molecular weights $(2,5,10 \mathrm{kDa})$ improved dispersion, neutralised charge and enhanced diffusion of poly(lactic-co-glycolic)acid (PLGA) in cervical mucus in a manner strongly dependent on the molecular weight and density of the polymer $(5-100 \%)$ on the particles surface. They revealed that diffusion of PEGylated PLGA nanoparticles was 3-10 times higher than that of uncoated PLGA nanoparticles.

PEGylated nanoparticles can also be used for oral drug delivery since they are able to diffuse across the mucus layer and thereby target specific regions for vaccination [33]. Yoncheva and coworkers reported the synthesis of poly(methyl vinyl ether-co-maleic anhydride) (PVM/MA) PEGylated nanoparticles, with PEG of different molecular weights (400, 1000 and $2000 \mathrm{Da})$ attached, for reaching the gastrointestinal mucosa. Particles were prepared by a solvent displacement method and PEGylation was found to be efficient with PEGs of molecular weight higher than 400 Da. Additionally, PEGylation with 2000 Da PEG was twice as effective as that of $1000 \mathrm{Da}$ and gave a "brush" conformation of PEG chains which enhanced penetration through the mucus layer and facilitated contact with the intestinal mucosa, illustrating their promise as carriers for drug delivery to the small intestine [33].

Among nanomaterials for biomedical application, silica nanoparticles exhibit great potential due to their straightforward synthesis and separation, low cost, safety, biocompatibility and possibility to further functionalization. PEGylation is a widely-employed approach to modify the surfaces of silica nanoparticles. Different levels of PEGylation can be achieved by varying the quantity of PEG added to the reaction mixture. Irmukhametova and coworkers [34] demonstrated that the presence of a PEG-based corona on the surface of NPs allows formation of hydrogenbonded interpolymer complexes with poly(acrylic acid) (PAA) under acidic conditions. In their study, selfassembly of PEGylated NPs with PAA occurred in aqueous solution and on silicon wafers. The first approach led to the formation of larger aggregates, while the second resulted in multilayered coatings.

Stability of PEGylated silica nanoparticle colloidal suspensions in water depends on the PEG/silica mass ratio. With appropriate PEG concentrations, surface modified silica NPs can remain stable to oxidation for up to 6 months [35]. In addition, PEGylation enhanced colloidal stability of silica nanoparticles over a wide range of pHs [34]. The colloidal stability of PEGylated silica nanoparticles in aqueous solutions can be improved by employing a grafting layers procedure [36]. To achieve water stability, a newly-developed approach utilised a two-step method of pre-coating silica nanoparticles with amino-groups (amination), followed by PEGylation with $N$-succinimidyl ester of a mono-methyl poly(ethylene glycol) carboxylic acid via covalent binding of $\mathrm{N}$-succinimidyl moieties to primary amino-groups. As an amino-source, a combination of (3-aminopropyl)triethoxysilane with bis-(trimethoxysilylpropyl)amine or bis-(triethoxysilyl)ethane was used, improving the water stability of PEGylated silica nanoparticles in aqueous dispersions. Additionally, this approach prevented nanoparticles from aggregation in the dry state [36]. By preventing aggregation, PEG also provides a homogeneous distribution of silica nanoparticles inside the poly( $\varepsilon$-caprolactone) (PCL) matrix, when acting as an interfacial agent in biodegradable PCL/PEG/silica nanocomposites. Increasing the content of PEGylated silica nanoparticles resulted in higher elastic moduli of these nanocomposites, as was confirmed by dynamic mechanical measurements [37]. 
Thiolated organosilica nanoparticles exhibited excellent mucoadhesive properties due to the number of SH groups present and can be modified to be nonadhesive via PEGylation [38]. Thiolated fluorescently-labelled nanoparticles were retained on the surface of bovine cornea even after five wash cycles with artificial tear fluid, whereas PEGylated nanoparticles were completely removed after the third wash. The ability of PEG to screen the functional groups on the surface of organosilica nanoparticles was further investigated by the same research group, providing a better understanding of the role of PEGylation in designing novel formulations [39-41]. Studying the retention of thiolated and PEGylated silica nanoparticles on the surface of porcine bladder mucosa in vitro demonstrated a more rapid wash-off profile of PEGylated silica nanoparticles than that of their parent particles indicating poorer mucoadhesive properties of PEGylated nanoparticles [41]. This was in agreement with the previously published data [38]. Poorer retention on the mucosal tissues of PEGylated nanoparticles arose an interest in studying their capability of permeating biological tissues due to the screening of functional groups of silica nanoparticles by PEG molecules and preventing them from interacting with the mucosal layers. That was investigated by assessing the capability of PEGylated silica nanoparticles to penetrate through the ocular surface [40]. It was shown that silica nanoparticles PEGylated with PEG of higher molecular weight (5000 Da) can penetrate de-epithelialized corneal surface into the stroma. This was due to the effective screening of thiol-groups and preventing them from interacting with cysteine domains of the ocular stroma [40]. These findings were in a agreement with the data reported by Hanes et al, revealing no interactions of PEGylated nanoparticles with biological tissues, such as human mucus, due to strong hydrophilicity and neutral charge provided by PEG molecules, helping the diffusion [31, 42]. Understanding the diffusion of PEGylated silica nanoparticles in complex fluids is essential in designing novel drug delivery formulations. Therefore, the diffusion of PEGylated silica nanoparticles was studied in polymer solutions of different chemical nature as prototypes of biological hydrogels [39]. It was demonstrated that PEGylation can greatly affect interactions between nanoparticles and the medium, hence the diffusion. In this way, PEGylated silica nanoparticles exhibited higher diffusivity in poly(ethylene oxide) solution than their thiolated counterpart due to no interactions between PEGylated surfaces and PEO macromolecules as a result of their chemical similarity. However, in polymers of a different nature, such as poly(acrylic acid) at lower $\mathrm{pH}$ values, PEGylation can significantly hamper the diffusion due to formation of strong hydrogen bonding between PEG moieties on the surface of nanoparticles and carboxyl groups of PAA [39].

PEGylated silica nanoparticles can be employed in the formation of blood pool contrast agent with encapsulated $\mathrm{Fe}_{3} \mathrm{O}_{4}$ nanocrystals. Wu and coworkers [43] demonstrated the advantages of a novel $\mathrm{H}-\mathrm{Fe}_{3} \mathrm{O}_{4} @ \mathrm{SiO}_{2}$-PEG agent for magnetic resonance imaging (MRI), reduced opsonisation and prolonged intravascular circulation due to the PEGylation. This provided an extended imaging window, enhanced vessel-to-background signal and minimised haemolytic activity of the nanoparticles. Due to these enhanced characteristics, $\mathrm{H}-\mathrm{Fe}_{3} \mathrm{O}_{4} @ \mathrm{SiO}_{2}-\mathrm{PEG}$ performed better than Resovist as demonstrated by recording excellent high-resolution images of rat cerebral microvasculature [43]. PEGylated fluorescein-doped magnetic silica nanoparticles can penetrate the blood-brain barrier (BBB), as was demonstrated in vivo by $\mathrm{Ku}$ et al. [44]. These nanoparticles were found to cross the BBB in rats via transcytosis of endothelial cells, followed by diffusion into cerebral parenchyma and subsequent distribution in the neurons. In addition, PEGylated magnetic silica nanoparticles did not show cumulative toxicity to the brain. However, their non-PEGylated counterpart was not found to penetrate the BBB. This demonstrates that PEGylated silica nanoparticles may offer a good platform for investigations of drug delivery systems and imaging in the treatment of brain diseases [44]. In intravenous drug delivery application of silica nanoparticles, PEGylation was employed to avoid the reticuloendothelial system. In addition, protein adsorption of silica nanoparticles was significantly decreased by PEGylation, and this reduction was constant with the PEG chain length of $3000 \mathrm{~g} / \mathrm{mol}$ [45].

PEGylation reduces the toxicity of mesoporous silica nanoparticles (MSNPs) which are of a considerable interest for drug delivery due to their facile synthesis, tunable pore morphologies, potential for further surface functionalisation and inclusion of various payloads [46]. PEGylated mesoporous silica nanoparticles can be formed using an emulsion method [47], sol-gel reaction [48], or facile one-pot synthesis in aqueous solution [49]. PEGylated MSNPs exhibit long- 
term stability in biological media, reduced aggregation and improved colloidal dispersity, showing potential benefits for drug delivery [49]. Singh and coworkers synthesised MSNPs coated with a polymer that degrades in response to external stimuli as temperature and biological microenvironment (protease). They proposed a new technique for mesoporous silica NPs double-coating through electrostatic binding of acrylamide with subsequent attachment of a covalently cross-linked PEG-based polymer shell, omitting the use of any catalysts or surfactants. PEGylated MSNPs displayed no significant in vitro cytotoxicity in HeLa cells, compared to uncoated MSNPs that were found to be cytotoxic. The polymer shell provided colloidal stability, prolonged blood circulation, was temperature sensitive, improved imaging capability, offered high core- and shell-loading capacities, generated localised drug release in vitro and in vivo and enabled tunability of drug loading and release characteristics [46].

Silica nanoparticles are widely used to deliver hydrophilic drugs, such as doxorubicin [50-52]. In the study of Xie et al. [52], the development of anticancer drug delivery and simultaneous bioimaging based on mesoporous silica nanoparticles was reported. MSNPs were functionalised with carboxyl groups to provide a more negative charge of the pore surface than that of the parent counterpart, which allowed binding through the attractive forces between silica nanoparticles and positively charged doxorubicin hydrochloride. PEGylation enabled conjugation of MSNPs with folate, as a cancer targeting moiety, and near infrared fluorescent dye, as a labelling agent. Additionally, introducing PEG to the nanoparticle structure increased their water dispersibility [52].

In targeted doxorubicin delivery to liver cancer cells, a new silane-free approach of functionalisation of mesoporous silica nanoparticles with a PEG layer was used [53]. This method involved the modification of the external surface of MSNPs with PEG and galactose ligands, while leaving the internal surface preserved for doxorubicin encapsulation. This was achieved by carrying out the two-step modification with surfactant remaining in the pore channels of MSNPs, which was removed upon completion of the functionalisation. PEG was introduced to prevent recognition of the drug delivery system as a foreign substance by the body defense mechanism. The PEG layer was also demonstrated to provide effective shielding of MSNPs from human serum protein adsorption. PEG-galactosemodified silica nanoparticles exhibited the maximum loading of doxorubicin at $900 \mathrm{mg} / \mathrm{g}$, revealed $\mathrm{pH}-$ dependent drug release and significantly higher cellular uptake, compared to pristine MSNPs. This demonstrated the clear potential of PEGylated mesoporous silica nanoparticles for intracellular delivery of anti-cancers drugs [53]. However, the delivery of hydrophobic anti-cancer drugs can also benefit from employing PEGylated silica nanoparticles. A layer of PEG conjugated phospholipids was shown to enhance the cell fusion capability and biocompatibility of silica nanoparticles for docetaxel delivery, protecting the drug from exposure to physiological fluids [54].

Porous silica NPs can also be modified by electrostatic self-assembly of polyethyleneiminepolyethylene glycol (PEI-PEG) copolymers onto their surfaces. Their biointerfacial properties can be improved by self-assembling PEI-PEG copolymers preventing biologically nonspecific adsorption events [55]. Surface modification with PEG can be effective for drug release control from hollow silica nanoparticles (HSNPs) with a large cavity, synthesised using magnetic assemblies as a template [56]. The authors demonstrated 10-times lower release rates of doxorubicin from the PEGylated HSNPs than from magnetic silica NPs due to the reduced size of the nanoparticle cavity as well as of the pores on silica wall. Additionally, burst release from the former was insignificant [56].

PEG molecular weight and chain density have an influence on nonspecific binding of PEGylated mesoporous silica nanoparticles to serum proteins and cellular responses [57]. It was shown that the optimal molecular weight of PEG was $>10 \mathrm{kDa}$ with optimal chain densities of 0.75 and $0.075 \mathrm{wt} \%$ for MSNPsPEG 10 and $20 \mathrm{kDa}$, respectively. The adsorbance of PEGylated silica nanoparticles to human serum albumin was revealed to be significantly lower than for bare MSNPs. Additionally, PEGylation provides the reduced percentage of human THP-1 phagocytosis and human red blood cells hemolysis, 0.1 and $0.9 \%$, respectively, in comparison to 8.6 and $14.2 \%$ of those of MSNPs [57]. PEGylation also affects biodegradation of colloidal mesoporous silica nanoparticles in simulated body fluid (SMF). It was shown that PEGylation reduces the degradation rate of silica nanoparticles in SMF at $37^{\circ} \mathrm{C}$, providing enhanced bio-stability, which is beneficial for developing injectable blood-persistent biomedical systems [58]. The stability and dispersity of hollow mesoporous silica nanoparticles (HMSNPs) in aqueous 
solutions were also improved by PEGylation [59]. The uptake of PEGylated hollow silica nanoparticles, synthesised by covalent attachment of poly(oxyethylene)bis(amine) to amino-functionalised hollow silica matrix, in HeLa and NIH3T3 cells was twice that of unmodified HMSNPs. In addition, PEGylated HMSNPs exhibited sustained release of doxorubicin hydrochloride as a model anticancer drug [59].

PEGylated silica nanoparticles found an application as a hybrid inorganic/organic system in achieving a constant release of large biological molecules over extended period of time [60]. PEG-coated mesoporous silica nanoparticles underwent hydrothermal treatment for pore expansion, followed by loading with a trypsin inhibitor. It was revealed that PEGylation prevents the initial burst release of protein, which was observed for uncoated MSNPs, and provides sustained release over several weeks through diffusion controlled release [60].

\section{Conclusion}

In summary, PEGylation is a useful tool for surface modification of nanoparticles, enhancing their properties and improving their functionality for drug delivery. PEGylation prevents nanoparticles from being recognised by the mononuclear phagocyte systems which prolongs their circulation in blood and allows drug delivery to the target tissue. In addition, PEGylation enhances solubility and stability of the nanoparticles, their penetrating ability through the mucus layer and makes the particles more compatible with the biological environment by converting hydrophobic surfaces of NPs to hydrophilic ones which reduces drug delivery time and toxicity. PEGylated nanoparticles offer a wide range of potential applications as drug carriers since they can provide safe and controlled delivery even for highly toxic anticancer agents such as doxorubicin.

\section{Acknowledgements}

The Science Committee of the Ministry of Education and Science of the Republic of Kazakhstan is acknowledged for funding this project (AP05135436, registration $\# 0118$ PK00908).

\section{References}

1. Zhang L., Gu F.X., Chan J.M., Wan A.Z., Langer R.S., Farokhzad O.C. Nanoparticles in medicine: Therapeutic applications and developments. Clinical
Pharmacology and Therapeutics, 2008, 83(5), 761-769. doi: 10.1038/sj.clpt.6100400

2. Jalali N., Moztarzadeh F., Mozafari M., Asgari S., Motevalian M., Alhosseini S.N. Surface modification of poly(lactide-co-glycolide) nanoparticles by $\mathrm{d}$ - $\alpha$-tocopheryl polyethylene glycol 1000 succinate as potential carrier for the delivery of drugs to the brain. Colloids Surfaces A: Physicochemical and Engineering Aspects, 2011, 392, 335-342. doi: 10.1016/j.colsurfa. 2011.10.012

3. Venkatpurwar V., Shiras, A., Pokharkar V. Porphyran capped gold nanoparticles as a novel carrier for delivery of anticancer drug: In vitro cytotoxicity study. International Journal of Pharmacetics, 2011, 409, 314-320. doi: 10.1016/j.ijpharm.2011.02.054

4. Canfarotta F., Whitcombe M.J. \& Piletsky S.A. Polymeric nanoparticles for optical sensing. Biotechnology Advances, 2013, 31, 1585-1599. doi: 10.1016/j.biotechadv.2013.08.010

5. Cheng Y., Morshed R.A., Auffinger B., Tobias A.L., Lesniak M.S. Multifunctional nanoparticles for brain tumor imaging and therapy. Advanced Drug Delivery Reviews, 2014, 66, 42-57. doi: 10.1016/j.addr. 2013.09.006

6. Jo J., Aoki I., Tabata, Y. Design of iron oxide nanoparticles with different sizes and surface charges for simple and efficient labeling of mesenchymal stem cells. Journal of Controlled Release, 2010, 142(3), 465-473. doi: 10.1016/j.jconrel.2009.11.014

7. Zhou C., Wang S., Zhou Y., Rong P., Chen Z., Liu J., Zhou J. Folate-conjugated $\mathrm{Fe}_{3} \mathrm{O}_{4}$ nanoparticles for in vivo tumor labeling. Transactions of Nonferrous Metals Society of China, 2013, 23(7), 2079-2084. doi: 10.1016/S1003-6326(13)62699-0

8. Fadeel B., Garcia-Bennett A.E. Better safe than sorry: Understanding the toxicological properties of inorganic nanoparticles manufactured for biomedical applications. Advanced Drug Delivery Reviews, 2010, 62, 362-374. doi: 10.1016/j.addr.2009

9. Bitar A., Ahmad N.M., Fessi H., Elaissari A. Silica-based nanoparticles for biomedical applications. Drug Discovery Today, 2012, 17, 1147-1154. doi: 10.1016/j.drudis.2012.06.014

10. Si S., Kotal A., Mandal T.K., Giri S., Nakamura H., Kohara T. Size-controlled synthesis of magnetite nanoparticles in the presence of polyelectrolytes. Chemistry of Materials, 2004, 16, 3489-3496. doi: 10.1021/cm049205n

11. Sun X., Zheng C., Zhang F., Yang Y., Wu G., Yu A., Guan N. Size-Controlled Synthesis of Magnetite $\left(\mathrm{Fe}_{3} \mathrm{O}_{4}\right)$ Nanoparticles Coated with Glucose and Gluconic Acid from a Single Fe ( III ) Precursor by a Sucrose Bifunctional Hydrothermal Method. Journal of Physical 
Chemistry C, 2009, 113,. 16002-16008. doi: 10.1021/jp9038682

12. Akamatsu K., Shimada M., Tsuruoka T., Nawafune H., Fujii S., Nakamura Y. Synthesis of pHResponsive Nanocomposite Microgels with SizeControlled Gold Nanoparticles from Ion-Doped, Lightly Cross-Linked Poly(vinylpyridine). Langmuir, 2010, 26, 1254-1259. doi: 10.1021/la902450c

13. Nakamura M., Ozaki S., Abe M., Doi H., Matsumoto T., Ishimura K. Size-controlled synthesis, surface functionalization, and biological applications of thiol-organosilica particles. Colloids Surfaces $B$ Biointerfaces, 2010, 79, 19-26. doi: 10.1016/j.colsurfb. 2010.03.008

14.Wang M., Löwik D.W.P.M., Miller A.D., Thanou M. Targeting the urokinase plasminogen activator receptor with synthetic self-assembly nanoparticles. Bioconjugate Chemistry, 2009, 20, 32-40. doi: 10.1021/bc8001908

15. Ghosh K., Kanapathipillai M., Korin N., McCarthy J.R., Ingber D.E. Polymeric nanomaterials for islet targeting and immunotherapeutic delivery. Nano Letters, 2012, 12, 203-208. doi: 10.1021/n1203334c

16. Nakamura M., Ishimura K. One-pot synthesis and characterization of three kinds of thiol - organosilica nanoparticles. Langmuir, 2008, 24, 5099-5108. doi: 10.1021/1a703395w

17.Toti U.S., Guru B.R., Grill A.E., Panyam J. Interfacial activity assisted surface functionalization: A novel approach to incorporate maleimide functional groups and cRGD peptide on polymeric nanoparticles for targeted drug delivery. Molecular Pharmaceutics, 2010, 7, 1108-1117. doi: 10.1021/mp900284c

18. Chen S., Li Y., Guo C., Wang J., Ma J., Liang X., Yang L.R., Liu H.Z. Temperature-responsive magnetite/ PEO-PPO-PEO block copolymer nanoparticles for controlled drug targeting delivery. Langmuir, 2007, 23, 12669-12676. doi: 10.1021/la702049d

19.Hu S.H., Liu T.Y., Huang H.Y., Liu D.M., Chen S.Y. Magnetic-Sensitive Silica Nanospheres for Controlled Drug Release. Langmuir, 2008, 24, 239-244. doi: 10.1021/la701570z

20.Ge J., Neofytou E., Cahill T.J., Beygui R.E., Zare R.N. Drug release from electric-field-responsive nanoparticles. ACS Nano, 2012, 6, 227-233. doi: 10.1021/nn203430m

21.Mohanraj V., Chen Y., Chen M. Nanoparticles A Review. Tropical Journal of Pharmaceuticsl Research, 2006, 5(1), 561-573. doi: 10.4314/tjpr.v5i1.14634

22. Owens D.E., Peppas N.A. Opsonization, biodistribution, and pharmacokinetics of polymeric nanoparticles. International Journal of Pharmaceutics, 2006, 307, 93-102. doi: 10.1016/j.ijpharm.2005.10.010
23.Li S., Huang L. Pharmacokinetics and Biodistribution of Nanoparticles. Molecular Pharmaceutics, 2008, 5, 496-504. doi: 10.1021/ mp800049w

24.Cheng Y., Samiya A.C., Meyers J.D., Panagopoulos I., Fei B., Burda C. Highly efficient drug delivery with gold nanoparticle vectors for in vivo photodynamic therapy of cancer. Journal of American Chemical Society, 2008, 130, 10643-10647. doi: 10.1021/ja801631c

25.Connor E.E., Mwamuka J., Gole A., Murphy C.J., Wyatt M.D. Gold nanoparticles are taken up by human cells but do not cause acute cytotoxicity. Small, 2005, 1, 325-327. doi: 10.1002/smll.200400093

26. Qian W., Murakami M., Ichikawa Y., Che, Y. Highly efficient and controllable PEGylation of gold nanoparticles prepared by femtosecond laser ablation in water. Journal of Physical Chemistry C, 2011, 115, 23293-23298. doi: 10.1021/jp2079567

27. Brown S.D., Nativo P., Smith J.A., Stirling D., Edwards P.R., Venugopal B., Flint D.J., Plumb J.A., Graham D., Wheate N.J. Gold Nanoparticles for the Improved Anticancer Drug Delivery of the Active Component of Oxaliplatin. Journal of American Chemical Society, 2010, 132, 4678-4684. doi: 10.1021/ja908117a

28. Craparo E.F., Cavallaro G., Bondi M.L., Mandracchia D., Giammona G. PEGylated nanoparticles based on a polyaspartamide. Preparation, physicochemical characterization, and intracellular uptake. Biomacromolecules, 2006, 7, 3083-3092. doi: 10.1021/ bm060570c

29.Gou M., Zheng X., Men K., Zhang J., Zheng L., Wang X., Luo F., Zhao Y., Zhao X., Wei Y., Qian Z. Poly( $\varepsilon$-caprolactone)/Poly(ethylene glycol)/Poly( $\varepsilon$ caprolactone) nanoparticles: preparation, characterization, and application in doxorubicin delivery. Journal of Physical Chemistry B, 2009, 113, 12928-12933. doi: 10.1021/jp905781g

30. Cu Y., Saltzman W.M. Controlled surface modification with poly(ethylene)glycol enhances diffusion of PLGA nanoparticles in human cervical mucus. Molecular Pharmaceutics, 2010, 6, 173-81. doi: 10.1021/mp8001254

31.Lai S.K., Wang Y.Y., Hanes J. Mucuspenetrating nanoparticles for drug and gene delivery to mucosal tissues. Advanced Drug Delivery Reviews, 2009, 61, 158-171. doi: 10.1016/j.addr.2008.11.002

32.Lai S.K., Suk J.S., Pace A., Wang Y.Y., Yang M., Mert O., Chen J., Kim J., Hanes J. Drug carrier nanoparticles that penetrate human chronic rhinosinusitis mucus. Biomaterials, 2011, 32, 6285-6290. doi: 10.1016/ j.biomaterials.2011.05.008 
33. Yoncheva K., Lizarraga E., Irache J.M. Pegylated nanoparticles based on poly(methyl vinyl ether-co-maleic anhydride): Preparation and evaluation of their bioadhesive properties. European Journal of Pharmaceutical Science, 2005, 24, 411-419. doi: 10.1016/j.ejps.2004.12.002

34. Irmukhametova G.S., Fraser B.J., Keddie J.L., Mun G.A., Khutoryanskiy V.V. Hydrogen-bonding-driven self-assembly of PEGylated organosilica nanoparticles with poly(acrylic acid) in aqueous solutions and in layerby-layer deposition at solid surfaces. Langmuir, 2012, 28, 299-306. doi: 10.1021/la2038735

35. Branda F., Silvestri B., Luciani G., Costantini A., Tescione F. Synthesis structure and stability of amino functionalized PEGylated silica nanoparticles. Colloids Surfaces A: Physicochemical and Engineering Aspects, 2010, 367, 12-16. doi: 10.1016/j.colsurfa.2010.05.036

36.Zhang Z., Berns A. E., Willbold S., Buitenhuis J. Synthesis of poly(ethylene glycol) (PEG)-grafted colloidal silica particles with improved stability in aqueous solvents. Journal of Colloid and Interface Science, 2007, 310, 446-455. doi: 10.1016/j.jcis.2007.02.024

37. Moussaif N., Irusta S., Yague C., Arruebo M., Meier J.G., Crespo C., Jimenez M.A., Santamaria J. Mechanically reinforced biodegradable nanocomposites. A facile synthesis based on PEGylated silica nanoparticles. Polymer, 2010, 51(26), 6132-6139. doi: 10.1016/j.polymer.2010.10.042

38. Irmukhametova G.S., Mun G.A., Khutoryanskiy V.V. Thiolated mucoadhesive and PEGylated nonmucoadhesive organosilica nanoparticles from 3-mercaptopropyltrimethoxysilane. Langmuir, 2011, 27, 9551-9556. doi: 10.1021/la201385h

39. Mun E.A., Hannell C., Rogers S.E., Hole P., Williams A.C., Khutoryanskiy V.V. On the role of specific interactions in the diffusion of nanoparticles in aqueous polymer solutions. Langmuir, 2014, 30, 308-317. doi: 10.1021/la4029035

40.Mun E.A., Morrison P.W.J., Williams A.C., Khutoryanskiy V.V. On the Barrier Properties of the Cornea: A Microscopy Study of the Penetration of Fluorescently Labeled Nanoparticles, Polymers, and Sodium Fluorescein. Molecular Pharmaceutics, 2014, 11, 3556-3564. doi: 10.1021/mp500332m

41.Mun E.A., Williams A.C., Khutoryanskiy V.V. Adhesion of thiolated silica nanoparticles to urinary bladder mucosa: Effects of PEGylation, thiol content and particle size. International Journal of Pharmaceutics, 2016, 512, 32-38. doi: 10.1016/j.ijpharm.2016.08.026

42.Xu Q., Boylan N.J., Cai S., Miao B., Patel H., Hanes J. Scalable method to produce biodegradable nanoparticles that rapidly penetrate human mucus.
Journal of Controlled Release, 2013, 170, 279-286. doi: 10.1016/j.jconrel.2013.05.035

43. Wu S.H., Lin C.Y., Hung Y., Chen W., Chang C., Mou C.Y. PEGylated silica nanoparticles encapsulating multiple magnetite nanocrystals for high-performance microscopic magnetic resonance angiography. Journal of Biomedical Materials Research Part B Applied Biomaterials, 2011, 99(1), 81-88. doi: 10.1002/ jbm.b.31874

44.Ku S., Yan F., Wang Y., Sun Y., Yang N., Ye L. The blood-brain barrier penetration and distribution of PEGylated fluorescein-doped magnetic silica nanoparticles in rat brain. Biochemical and Biophysical Research Communications, 2010, 394(4), 871-876. doi: 10.1016/j.bbrc.2010.03.006

45. Yagüe C., Moros M., Grazú V., Arruebo M., Santamaría J. Synthesis and stealthing study of bare and PEGylated silica micro- and nanoparticles as potential drug-delivery vectors. Chemical Engineering Journal, 2008, 137(1), 45-53. doi: 10.1016/j.cej.2007.07.088

46. Singh N., Karambelkar A., Gu L., Lin K., Miller J.S., Chen C.S., Sailor M.J., Bhatia S.N. Bioresponsive Mesoporous Silica Nanoparticles for Triggered Drug Release Bioresponsive Mesoporous Silica Nanoparticles for Triggered Drug Release. Journal of American Chemical Society, 2011, 133, 19582-19585. doi: $10.1021 / \mathrm{ja} 206998 \mathrm{x}$

47.Oh C., Ki C.D., Chang J.Y., Oh S. G. Preparation of PEG-grafted silica particles using emulsion method. Materials Letters, 2005, 59, 929-933. doi: 10.1016/ j.matlet.2004.09.048

48. Oh C., Lee Y.G., Choi T.S., Jon C.U., Oh S.G. Facile synthesis of PEG-silica hybrid particles using onestep sol-gel reaction in aqueous solution. Colloids Surfaces A Physicochemical Engineering Aspects, 2009, 349, 145-150. doi: 10.1016/j.colsurfa.2009.08.008

49.Zhang Q., Ye Z., Wang S.T., Yin J. Facile onepot synthesis of PEGylated monodisperse mesoporous silica nanoparticles with controllable particle sizes. Chinese Chemical Letters, 2014, 25, 257-260.

50.Chen Y., Yang W., Chang B., Hu H., Fang X., Sha $\mathrm{X}$. In vivo distribution and antitumor activity of doxorubicin-loaded $\quad \mathrm{N}$-isopropylacrylamide-comethacrylic acid coated mesoporous silica nanoparticles and safety evaluation. European Journal of Pharmaceutics and Biopharmaceutics, 2013, 85, 406-412. doi: 10.1016/j.ejpb.2013.06.015

51.Cui Y., Xu Q., Chow P.K.H., Wang D., Wang C.H. Transferrin-conjugated magnetic silica PLGA nanoparticles loaded with doxorubicin and paclitaxel for brain glioma treatment. Biomaterials, 2013, 34, 85118520. doi: 10.1016/j.biomaterials.2013.07.075 
52.Xie M., Shi H., Li Z., Shen H., Ma K., Li B., Shen S., Jin Y. A multifunctional mesoporous silica nanocomposite for targeted delivery, controlled release of doxorubicin and bioimaging. Colloids Surfaces $B$ Biointerfaces, 2013, 110, 138-147. doi: 10.1016/ j.colsurfb.2013.04.009

53.Gu J., Su S., Zhu M., Li Y., Zhao W., Duan Y., Shi J. Targeted doxorubicin delivery to liver cancer cells by PEGylated mesoporous silica nanoparticles with a pH-dependent release profile. Microporous Mesoporous Materials, 2012, 161, 160-167. doi: 10.1016/ j.micromeso.2012.05.035

54.Liu Y., Mi Y., Zhao J., Feng S.S. Multifunctional silica nanoparticles for targeted delivery of hydrophobic imaging and therapeutic agents. International Journal of Pharmaceutics, 2011, 421, 370-378. doi: 10.1016/ j.ijpharm.2011.10.004

55. Thierry B., Zimmer L., McNiven S., Finnie K., Barbe C., Griesser H.J. Electrostatic self-assembly of PEG copolymers onto porous silica nanoparticles. Langmuir, 2008, 24, 8143-8150. doi: 10.1021/la8007206

56. Yang J., Lee J., Kang J., Lee K., Suh J.S., Yoon H.G., Huh Y.M., Haam S. Hollow silica nanocontainers as drug delivery vehicles. Langmuir, 2008, 24, 3417-3421. doi: 10.1021/la701688t

57.He Q., Zhang J., Shi J., Zhu Z., Zhang L., Bu W., Guo L., Chen Y. The effect of PEGylation of mesoporous silica nanoparticles on nonspecific binding of serum proteins and cellular responses. Biomaterials, 2010, 31, 1085-1092. doi: 10.1016/j.biomaterials.2009.10.046

58. Cauda V., Schlossbauer A., Bein T. Biodegradation study of colloidal mesoporous silica nanoparticles: Effect of surface functionalization with organo-silanes and poly(ethylene glycol). Microporous and Mesoporous Materials, 2010, 132, 60-71. doi: 10.1016/j.micromeso.2009.11.015

59.Zhu Y., Fang Y., Borchardt L., Kaskel S. PEGylated hollow mesoporous silica nanoparticles as potential drug delivery vehicles. Microporous and Mesoporous Materials, 2011, 141, 199-206. doi: 10.1016/j.micromeso.2010.11.013

60. Bhattacharyya S., Wang H., Ducheyne P. Polymer-coated mesoporous silica nanoparticles for the controlled release of macromolecules. Acta Biomaterials, 2012, 8, 3429-3435. doi: 10.1016/j.actbio.2012.06.003 